\title{
Nanotechnology for the suppression of fires in agricultural land and forests
}

\author{
Valery Belozerov ${ }^{1, *}$, Mihail Nikulin ${ }^{2}$ and Nikolay Topolsky ${ }^{3}$ \\ ${ }^{1}$ Don State Technical University, 1, sq. Gagarina, 344003, Rostov-on-Don, Russia \\ ${ }^{2}$ State Agrarian University of the Northern Trans-Urals ", 7, Republic St., 625003, Tyumen, Russia \\ ${ }^{3}$ Academy of State Fire Service, 4, st. B. Galushkina, 129366, Moscow, Russia
}

\begin{abstract}
The article presents the results of a systematic analysis of the causes and consequences of forest and landscape fires in the USSR and in Russia, which indicate the lack of effective fire protection methods and means from them to date. It is shown that the world community, in addition to flooding landscape and forest fires, bunding and filling of their distribution paths with earth, has no other ways and means of suppressing them. The results of research and synthesis of nanotechnologies and models of fire-fighting helicopters are presented, in which the planet's atmosphere is used as an "endless source of fire extinguishing composition", which allows using the developed (thermomagnetic air separators) and produced (membrane air separators and helicopters) reequip «Avialesookhrana» and the Ministry of Emergencies of Russia in order to reverse the growth trends of fires and losses from them in the forests and steppes of Russia. It is noted that the proposed nanotechnology for suppressing fire can be effectively used not only to extinguish fires on farmland and forests, but also on trunk oil and gas pipelines, vehicles on roads (away from settlements) and facilities in the mountains where water and other extinguishing agent delivery options are limited.
\end{abstract}

\section{Introduction}

Russia, which accounts for one fifth of all the world's forests, is considered a leading forest power, with a forest area of 8.1 million square kilometers, which occupies $49.4 \%$ of the country. Canada holds the second place - 4.9 million square kilometers and $49.2 \%$ of the territory, respectively. Then follow Brazil -4.7 million square kilometers $(56.1 \%)$, the United States - 3.1 million square kilometers (33.8\%), China - 2.1 million square kilometers $(21.8 \%)$ and Australia - 1.3 million square kilometers (16.0\%).

The post-war statistics of forest fires in the USSR (Fig. 1.2) indicates that 20 to 30 thousand forest fires occurred annually in the country on an area of 0.5 to 2.5 million ha, and today only in Siberian forests occurs up to 27 thousand fires, which cover an area of 3.5 to 18 million hectares. [1,2].

\footnotetext{
*Corresponding author: safeting@mail.ru
} 
As studies of Russian scientists have shown, only in $10 \%$ of cases the cause of forest fires is climatic disasters, and the real extent of forest fires in Russia and the actual damage from destruction by fire have not been reliably established to date $[2,3]$.

The fight against landscape fires, in addition to the complexity of their localization and elimination, require huge costs. The cost of extinguishing one major fire can amount to tens of millions of rubles, and socio-economic losses - to hundreds of billions! Therefore, many countries, such as the USA, China, Canada, Australia and Russia, for which the problem of forest fires is relevant, have special aviation fire units $[1,4,5]$.

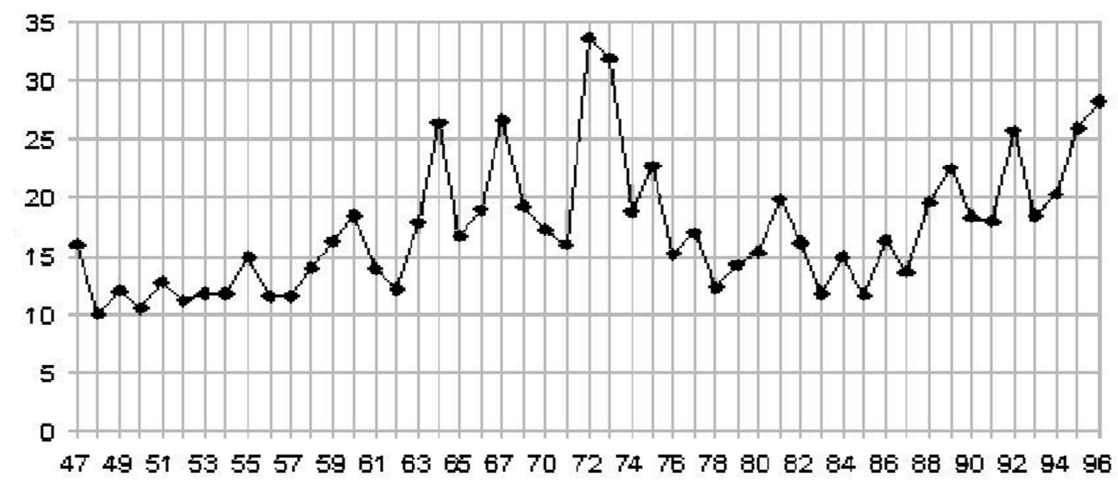

Fig. 1. Statistics of forest fires in the USSR / Russia in 1947-1996 (thousand fires).

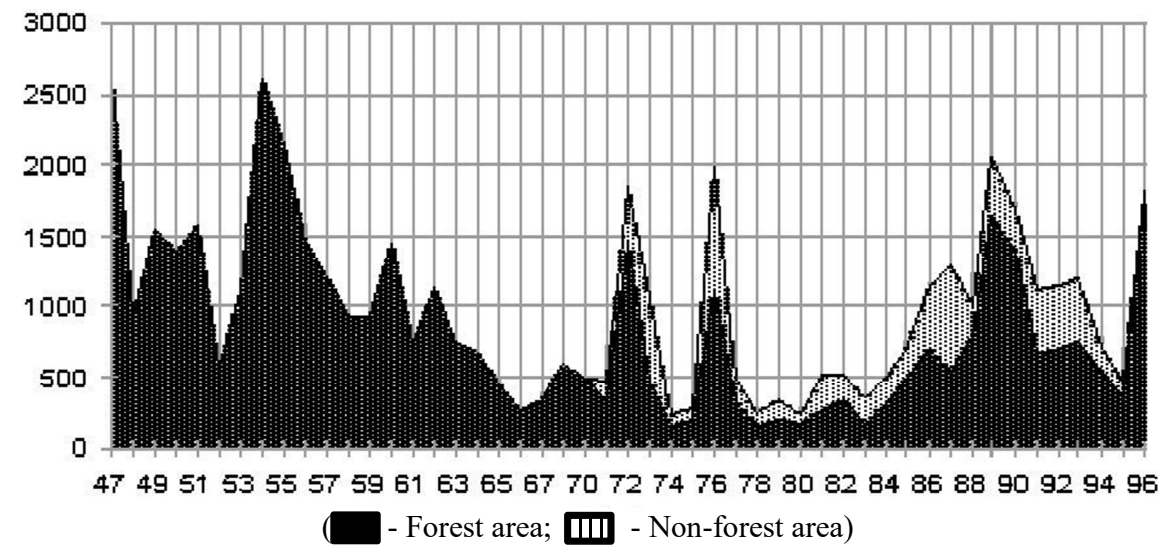

Fig. 2. Statistics of forest fire areas in the USSR in 1947-1996 (thousand hectares).

\section{Results and discussion}

In the USSR, the fire fighting service as part of the forestry department has been operating since the beginning of the 30 s of the last century and until now disputes about the effectiveness of its work have not ceased. However, in the early 90s, the situation changed dramatically, because constant underfunding, redistribution of ownership in the aviation industry, and reforms in the forestry department have led to a decrease in the efficiency of the "expensive" fire-fighting service $[1,4]$.

The consequence of this attitude towards aviation protection was a sharp deterioration in the results of its activities and a catastrophic decrease in the level of fire protection of forests, because its contribution to the detection and extinguishing of forest fires was reduced by almost 2 times. At the same time, the ground forest guard service, insufficiently equipped with fire fighting equipment, transport and communications, could not cope with 
the sharply increased volumes of fire fighting, resulting in an increase in the number of forest fires that take on the nature of natural disasters $[1,2,5]$.

Currently, several types of fire helicopters and airplanes are produced in Russia, many of which are in service with the Russian Emergencies Ministry, including to extinguish forest and steppe fires $[1,4]$ :

Central grouping: IL-76P, IL-62, Yak-42, Be-200ChS, Mi-26, Mi-8, Bo-105, Bk-117;

Southern grouping: An-3, Ka-32A1, Mi-8;

Siberian group: An-74, An-3, Mi-26, Mi-8;

Far Eastern grouping: An-74, Be-200ES, Mi-26, Mi-8.

To date, not a single country has any new methods and means of dealing with forest fires, other than flood them with water, land debris and fall asleep. However, extinguishing forest and steppe fires with water using aviation is not only economically unjustified, but also not effective, since airplanes and helicopters constantly have to refuel, fly up to the fire site, pour water and fly away to refuel, during which time the fire flares up new power $[1,5]$.

A comparative analysis of the use of existing fire-fighting aircraft and helicopters to extinguish landscape and forest fires showed (Table 1) the following systemic differences in their application.

Table 1. The tactical and technical data of MI-8 with APU-5 and Be-200 amphibious aircraft.

\begin{tabular}{|l|c|c|c|c|c|}
\hline \multicolumn{1}{|c|}{ Aircraft } & Depth of & The width of & \multirow{2}{*}{$\begin{array}{c}\text { The volume } \\
\text { the } \\
\text { reservoir, } \\
\mathbf{m}\end{array}$} & $\begin{array}{c}\text { The reservoir, } \\
\mathbf{m}\end{array}$ & \multicolumn{2}{|c|}{$\begin{array}{c}\text { Delivery speed, } \mathbf{k m} / \mathbf{h} \\
\text { oubic meter }\end{array}$} & $\begin{array}{c}\text { water } \\
\text { tanks }\end{array}$ & $\begin{array}{c}\text { containers } \\
\text { without } \\
\text { water }\end{array}$ \\
\hline $\begin{array}{l}\text { MI-8 c spillway } \\
\text { devices (SWD-5) }\end{array}$ & $0.7-1$ & unlimited & $3-4.5$ & 260 & 400 \\
\hline Be-200 & 2.5 & 2300 & 12 & 600 & 710 \\
\hline
\end{tabular}

Today, the most effective ways to deal with fires using Be-200 fire-fighting amphibious aircraft are [5]:

- laying fire barriers and support strips on flat terrain in order to stop the spread of combustion until the approach of ground forces and fire extinguishing means;

- reduction in the intensity of combustion in the most straightforward sections of the edge of the fire (edge quenching) in order to create conditions for ground forces to switch from an indirect method of extinguishing to direct;

- air patrolling for early detection of fire sources.

Unlike an amphibious aircraft, in a MI-8 helicopter with a 5-ton spillway devices (SWD-5), the speed of transporting a container with water is much lower, and in case of fires in small territories or in mountainous areas this is a fundamental advantage, since when draining at high speeds (exceeding $250 \mathrm{~km} / \mathrm{h}$ ), at altitudes exceeding $40-50 \mathrm{~m}$ from the surface of the earth, the discharged liquid breaks up as a result of an oncoming air flow to the state of aerosols and most of it evaporates before reaching the fire source [5].

Thus, helicopters including drones can become the main technical means in the modern system of fire protection of forests and steppes, and it remains to equip them with effective fire extinguishing compounds and the latest fire detection tools.

Atmospheric nitrogen is beyond competition in the ability to extinguish any fires [6], because It does not damage buildings, nor metal structures, nor electrical and radioelectronic equipment, nor books, nor furniture, nor other cultural values and household items, nor does it harm living organisms (humans, farm animals, etc.), in connection with than, it has long been used in gas volumetric fire extinguishing systems. However, fire tactics for local fire extinguishing with gaseous nitrogen do not exist either in Russia or abroad. Nevertheless, a number of research projects carried out by Russian scientists have 
proved the possibility of extinguishing fires with nitrogen (Fig. 3) obtained from air using membrane installations (Fig. 4), including manufactured in Russia [7.8].

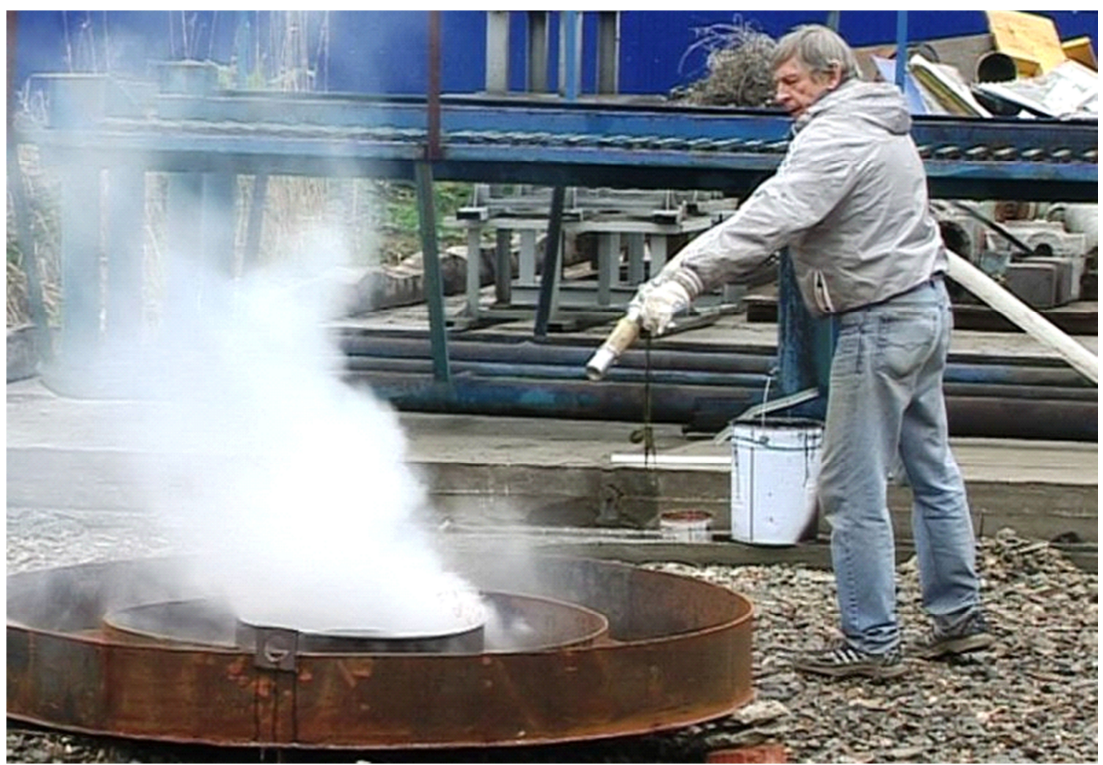

Fig. 3. Extinguishing a standard fire with nitrogen from a TGA membrane unit.

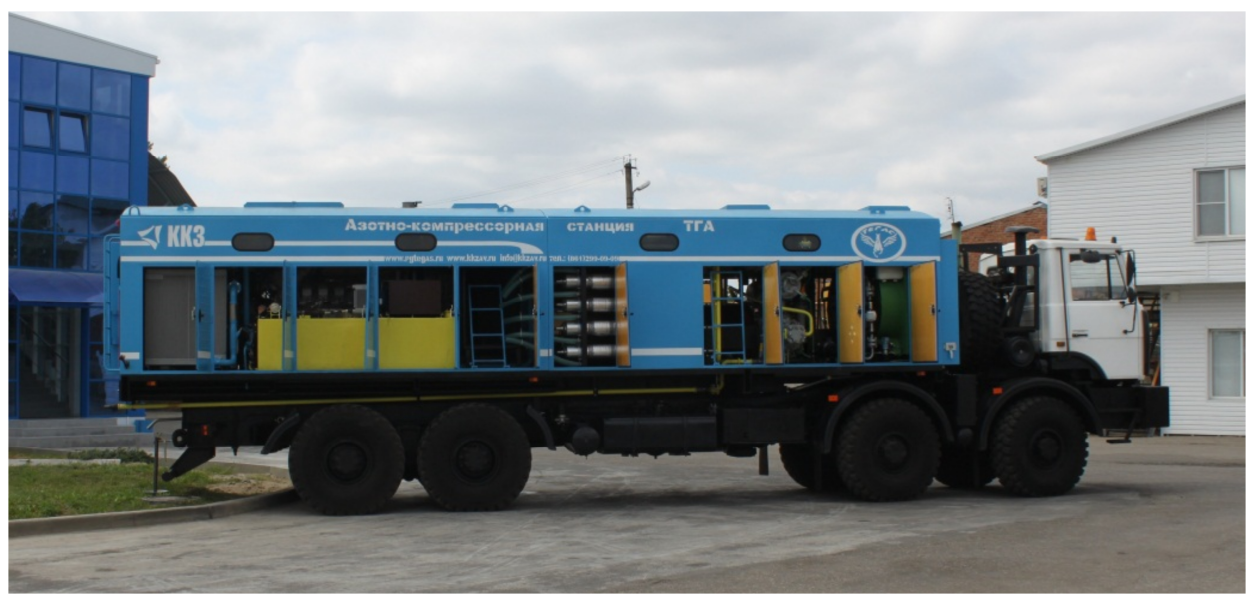

Fig. 4. TGA $20 \backslash 251$ C95 mobile compressor station on the MZKT chassis.

All currently manufactured membrane separators of air (MSA) are built on the principle of Knudsen diffusion, according to which the components of the mixture to be separated penetrate the pores of the membrane at different speeds, and therefore, the separation coefficient of the mixture depends on molecular weights $[9,10]$ :

$$
\mathrm{Cr}=\mathrm{n}_{1} / \mathrm{n}_{2}=-\left(\mathrm{M}_{2} / \mathrm{M}_{1}\right)^{0.5} \text {, }
$$

where $\mathrm{n}_{1}$ and $\mathrm{n}_{2}$ are the numbers of moles of components, respectively, with molecular weights $\mathrm{M}_{1}$ and $\mathrm{M}_{2}$.

The TGA-type nitrogen compressor stations manufactured by the Krasnodar Compressor Plant have introduced the latest technical solutions and equipment that 
significantly improve the station's performance in terms of convenience and reliability, in comparison with domestic and foreign competitors, namely [10,11]:

- increased nitrogen purity at the outlet, adjustable from 95 to $99.99 \%$ :

- the productivity of the stations is from 0.1 to 1 cubic meter of nitrogen per second;

- nitrogen pressure at the outlet of the station is possible in the range from 5 to 400 at.;

- implemented the ability to regulate performance from 50 to $100 \%$;

- microprocessor automation system provides the ability to control with remote access;

- the modernized cooling system of the diesel engine and compressor allows the station to work with higher (lower) ambient temperatures;

- increased compressor service life up to 5000 hours or more;

- increased fuel tanks from 800 to 1200 liters (24 hours);

- introduced automatic steam traps;

- ensured stable operation at lower temperatures $\left(-60^{\circ} \mathrm{C}\right)$.

Thus, the idea "to suspend a container with TGA" to the Mi-26T helicopter instead of spillway devices (Fig. 5) appeared. And in this case, it was proposed $[8,10]$ to supplement the TGA container (Fig. 6) with a "receiver battery with Laval nozzles" designed for synchronous and supersonic supply of nitrogen separated from air into the fire below it at a distance of 10 to 50 meters .
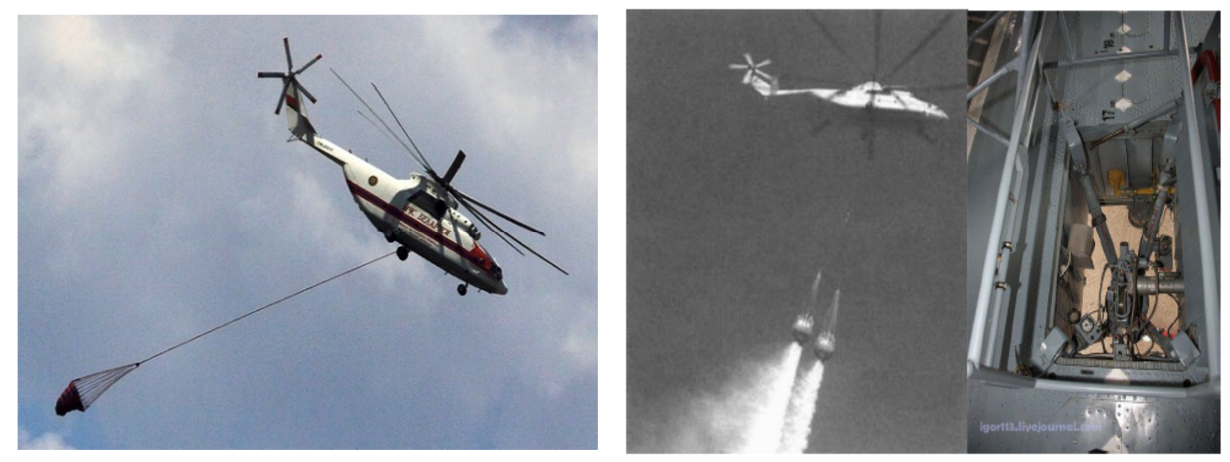

Fig. 5. Mi-26T with SWD-15 and with two "bambi-buckets" with an external suspension unit.
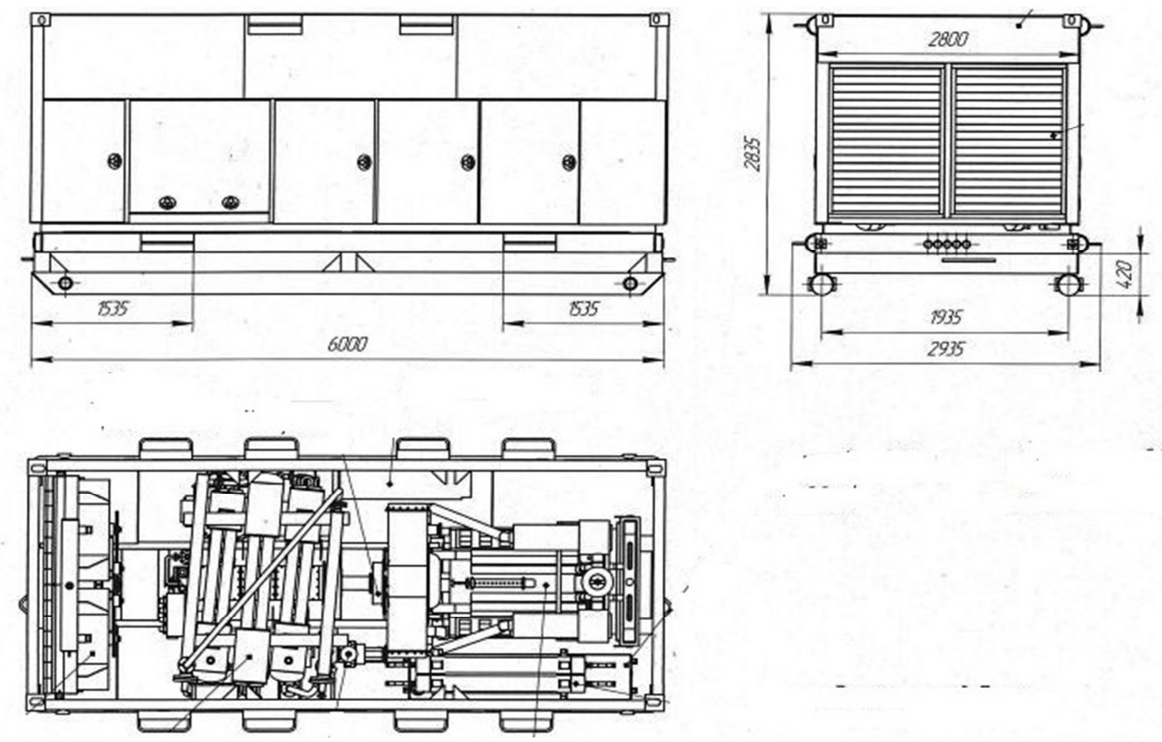

Fig. 6. Hanging container nitrogen station NDA-10/251. 
Another solution was proposed $[1,8,13]$ for extinguishing steppe and landscape fires with low forest undergrowth and shrubs with the Mi-8 helicopter (Fig. 7).

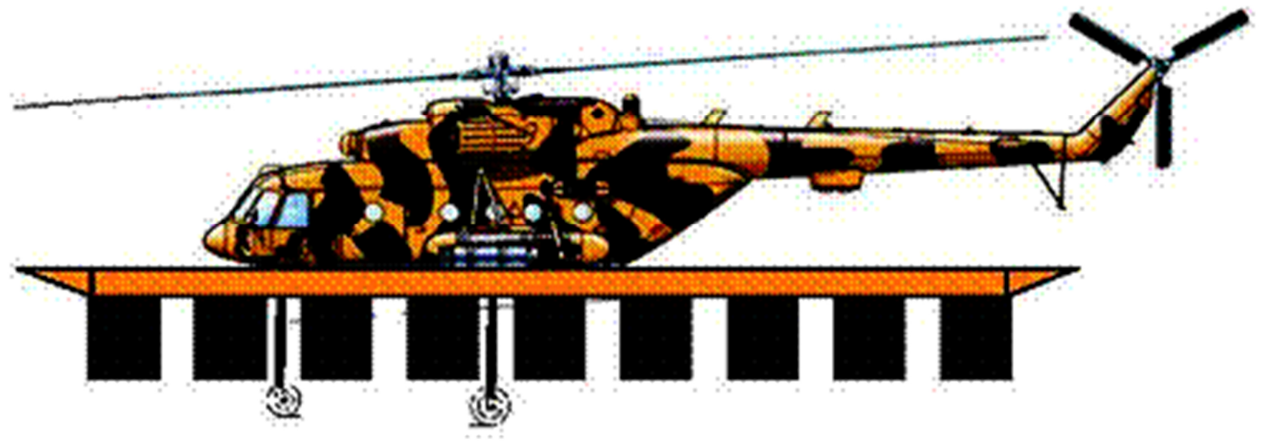

Fig. 7. Model of a fire helicopter with thermomagnetic air separators.

Rostov scientists developed a method of thermomagnetic separation of the air flow (TMSA) in a decreasing transverse magnetic field into oxygen (paramagnetic) and nitrogenwith other gases (diamagnetic) and a separator model that implements it $[12,13]$ :

$$
\rho\left(\frac{\partial \vec{v}}{\partial t}+(\vec{v} \cdot \nabla) \vec{v}\right)=-\operatorname{grad}(p)-\rho \cdot \operatorname{grad}\left(-\frac{\alpha H^{2}}{2 m}\right)
$$

Substituting the equation of state of an ideal gas $\mathrm{pV}=\mathrm{NkT}$ into Euler's equation (2), and expressing the density of the gas through its pressure $\mathrm{P}=\mathrm{nkT}=\rho \mathrm{kT} / \mathrm{m}$, an expression for the density of gas molecules was obtained in the form of the Boltzmann distribution

$$
\rho=\rho_{0} \exp \left(\frac{\alpha H^{2}}{2 k T}\right)=\rho_{0} \exp \left(-\frac{U}{k T}\right)
$$

where $\mathrm{U}=-\alpha \mathrm{H}^{2} / 2$ is the potential energy of an individual gas molecule with para- or diamagnetic properties, located in an external inhomogeneous magnetic field.

For oxygen $\left(\mathrm{O}_{2}\right)$, which has paramagnetic properties, the average magnetic polarizability of an individual molecule is positive $\left(+3396 \cdot 10^{-6}\right)$, and for nitrogen $(-12$. $10^{-6}$ ) and other gases with diamagnetic properties are negative. Therefore, the oxygen density increases at the wall with permanent magnets, i.e. in the region of a strong magnetic field, in accordance with equation (3), and the density of the nitrogen component decreases, depending on the square of the magnetic field inside the separator channel (Fig. 8). Moreover, as follows from the Boltzmann distribution (3), the separation effect is accelerated if the opposite wall is cooled, i.e. the region of a weak magnetic field where diamagnets are "pushed" $[12,13]$. 

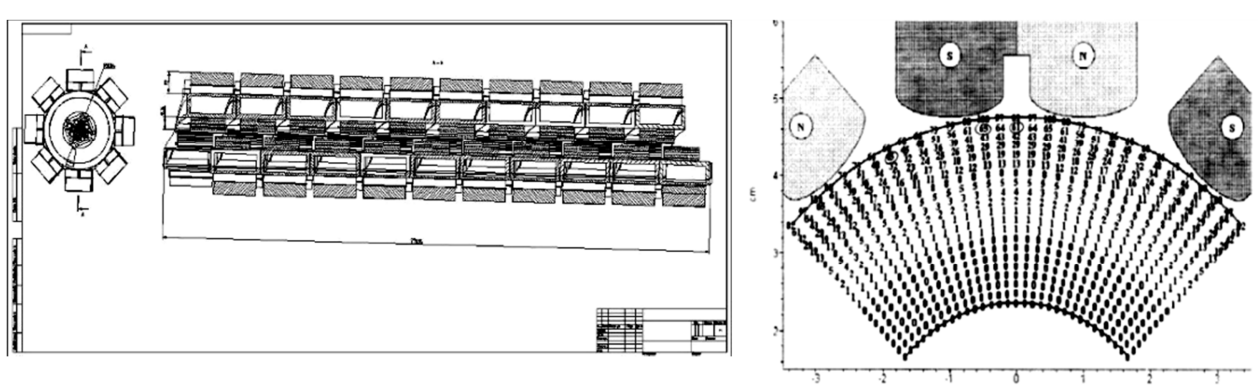

Fig. 8. Turn-on TMSA and distribution of the magnetic field vector (in \%).

To suppress the backward diffusion of oxygen, a solid porous aluminum baffle is installed along the TMSA channel (Fig. 9), which functions as an oxygen membrane at a distance that divides the volume of flowing air in a ratio of 1:4 from the side with permanent magnets, then at the exit "paramagnetic subchannel" we get pure oxygen, and at the output of the "diamagnetic subchannel"- nitrogen with other atmospheric gases $[14,15]$.

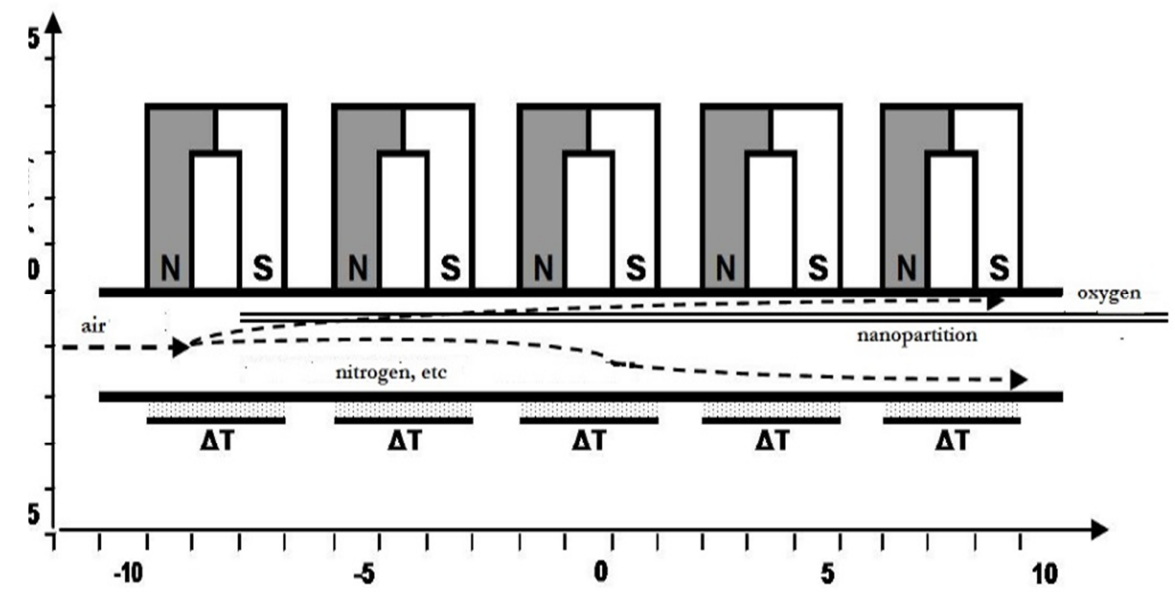

Fig. 9. Block diagram of a thermomagnetic air separator (TMSA).

Thus, from the atmosphere of the planet we get "an endless source of fire extinguishing composition" (ESFEC), consisting of:

$-98.77 \%$ nitrogen,

$-1.17 \%$ argon,

$-0.37 \%$ carbon dioxide,

$-0.01 \%$ helium,

which, individually, have long and successfully been used in various gas fire extinguishing installations and vehicles. Therefore, it remains to determine the necessary diamagnetics feed rate and develop fire tactics for local fire extinguishing with their help [7,8].

MI-8, creates air pressure under the screw up to $40 \mathrm{~atm}$., at an air flow rate of up to 20 $\mathrm{m} / \mathrm{s}$. If you place a "mounted installation" of thermomagnetic air separators under the bottom of the helicopter (by the diameter of the screw), then you can "intercept and turn" the entire air stream from the screw into a stream of "cooled diamagnets", which extinguish the fire, barraging burning areas, and freezing above the foci as long as the helicopter has fuel. In this case, the "discharge" of oxygen is carried out up the perimeter of the "mounted installation, which prevents surging of the engine due to combustion products [8].

Cooling of "diamagnetic" (at the outlet it can reach a temperature of minus $40^{\circ} \mathrm{C}$ ) is carried out by Azarov vortex modules [13,15] from the same air flow of the propeller, 
which will allow a fire helicopter not only to localize the steppe fire, but also to completely eliminate it, since the flow of "diamagnetic", when the helicopter hangs over the fire, it will not only extinguish the flame, isolating the burning vegetation from atmospheric oxygen, but also cool the perimeter of the fire, thereby allowing the use of the boning technique, cutting off the fire from the rest of the array.

As numerous studies have shown, to date, no country in the world has a satisfactory solution to the problem of fire protection from forest and steppe fires, in connection with which, the proposed use of nanotechnologies for gas separation of atmospheric air and arming them with installations that implement MI-26 and MI-8 helicopters, according to the authors, it will allow to solve the most important problem - fire protection of forest and steppe areas of Russia, as well as export methodological and technical equipment to other countries of the world $[16,17]$

\section{Conclusion}

As shown by numerous studies, no country in the world has a satisfactory solution to the problem of fire protection from forest and steppe fires to date.

It should be noted that in case of successful completion of work on the creation and development of the production of TMSA and "suspended stations MSA", fire helicopters with ESFEC, together with existing ground equipment, will not only be able to completely solve the problems of detection and suppression of forest and steppe fires, but also be effectively used when extinguishing fires on main oil and gas pipelines, vehicles on roads (away from settlements), and objects in the mountains where water supply is limited.

\section{References}

1. N.G. Topolsky, V.V. Belozerov, N.S. Afanasyev, Technologies of technosphere safety 4(32), 6 (2010) http://academygps.ucoz.ru/ttb/2010-4/2010-4.html

2. P.A. Tsvetkov, L.V. Buryak, Siberian Forest Journal 3, 25-42 (2014)

3. A.V. Volokitina, D.I. Nazimova, T.M. Sofronova, M.A. Korets, Results and Prospects of Geobotanical Research in Siberia: Russian Scientific-Practical conference dedicated to the 75th anniversary of the laboratory of ecology and geobotany of the $C S B G \quad S B \quad R A S$ (Academic publishing house "Geo" ,Novosibirsk, 2019) http://www.csbg.nsc.ru/uploads/confer/2019/Abstracta_book.pdf.

4. F.A. Kurakov, Economics of Science 3(3), 214-226 (2017) DOI 10.22394 / 2410132X-2017-3-3-214-226

5. A.O. Grigoryevskaya, N.V. Ivanov, A.V. Vishnev, Reshetnevsky readings: collection XVIII Intern. scientific Conf. Acad. 90th Birthday M.F. Reshetneva. Part 1 (SibSAU, Krasnoyarsk, 2014)

6. V.P. Nazarov, A.A. Kornilov, Technosphere safety technologies 4(32), 7 (2010) http://academygps.ucoz.ru/ttb/2010-4/2010-4.html

7. A. Lukyanov et al., Sat Proceedings of the Scientific and Practical Conference (LLC Krasnodar Compressor Plant, Krasnodar, 2013) https://www.elibrary.ru/item.asp?id=28186582

8. I.V. Voroshilov, B.Ch. Meskhi, A.I. Prilutsky, Electronics and electrical engineering 1, 21 - 71 (2016) DOI: 10.7256 / 2453-8884.2016.1.21034.

9. I.V. Voroshilov, G.I. Maltsev, A.Yu. Koshakov, Nitrogen generator, Patent of the Russian Federation No. 02450857 (2012) 
10. I.V. Voroshilov and others, Drilling and Oil 5, 64-65 (2012)

11. I.V. Voroshilov and other, Gas industry 9, 84-85 (2012)

12. V.V. Belozerov, S.I. Bosy, Yu.A. Videtskikh, A.A. Novakovich, M.G. Pirogov, G.N. Tolmachev, The method of thermomagnetic air separation and device for its implementation, Patent of the Russian Federation No. 2428242 (2011)

13. V.G. Zakharevich, G.G. Matishov, V.I. Shumeyko, Electronics and electrical engineering 1, 16 - 44 (2017) DOI: 10.7256 / 2453-8884.2017.1.21781

14. L.Kh. Badalyan, V.N. Kurdyukov, Development of a methodology for assessing atmospheric air pollution and reducing damage to the city's ecosystem (DSTU, Rostov-on-Don, 2015)

15. A.I. Azarov, Vortex tubes in industry. Inventor - mechanical engineering. Energy conservation and vortex effect: research and development of innovative projects (Publishing house LEMA, St. Petersburg, 2010)

16. V.V. Belozerov, I.V. Voroshilov, O.V. Zimovnov, M.A. Nikulin, P.S. Obukhov, Vl. V. Belozerov, A method for detecting, preventing the spread of fire and extinguishing forest fires with atmospheric nitrogen using a helicopter, application for invention No. 2020107085 (2020)

17. D.V. Abrosimov, V.V. Belozerov, O.V. Zimovnov, M.A. Nikulin, M.N. Filimonov, V1.V. Belozerov, A method for detecting and extinguishing helicopter landscape fires with inert atmospheric gases, application for invention No. 2020107241 (2020) 